\title{
Prognostic analysis and predictive rule for outcome of hospital-treated community-acquired pneumonia
}

\author{
S. Ewig, T. Bauer, E. Hasper, L. Pizzulli, R. Kubini, B. Lüderitz
}

Prognostic analysis and predictive rule for outcome of hospital-treated communityacquired pneumonia. S. Ewig, T. Bauer, E. Hasper, L. Pizzulli, R. Kubini, B. Lüderitz. (C)ERS Journals Ltd 1995.

ABSTRACT: In community-acquired pneumonia (CAP) mortality may be reduced by early identification of patients requiring intensive care treatment. The purpose of this study was to determine prognostic factors of outcome in patients with CAP in order to establish a clinically applicable discriminant rule.

Ninety three episodes of CAP in 92 patients were retrospectively reviewed with regard to epidemiological, clinical, laboratory and microbiological data. The prognostic analysis included a univariate as well as a multivariate approach, in order to identify parameters associated with death using the Cox regression hazard function in a backward stepwise selection model. The three parameters found to contribute most to the significance of the model were used in a discriminant rule for classification of outcome.

The parameters found to be significantly different between survivors and nonsurvivors were heart rate, systolic and diastolic as well as mean blood pressures, leucocyte count, percentage of lymphocytes, and lactate dehydrogenase (LDH) values. The multivariate analysis revealed that heart rate, systolic arterial pressure, and LDH serum levels were most closely associated with fatal outcome. A prognostic rule composed of the variables heart rate $\geq 90$ beats $\cdot \mathrm{min}^{-1}$, systolic arterial blood pressure $\leq 80 \mathrm{mmHg}$, and $\mathrm{LDH} \geq 260 \mathrm{U} \cdot l^{-1}$ achieved a sensitivity of $77 \%$, a specificity of $75 \%$, and positive and negative predictive values of 42 and $93 \%$, respectively. It was associated with a six fold increased risk of fatal outcome.

In conclusion, heart rate, systolic blood pressure, and LDH values were most closely associated with death in a multivariate analysis. A discriminant rule consisting of these three variables achieved favourable classification results. The rule qualifies for further prospective validation, and may prove useful in the management of hospital-treated CAP.

Eur Respir J., 1995, 8, 392-397.
Dept of Medicine, University of Bonn,
Bonn, Germany.

Correspondence: S. Ewig

Medizinische Universitätsklinik und

Poliklinik Bonn

Innere Medizin/Kardiologie und

Pneumologie

Sigmund Freud-Str.25

53105 Bonn

Germany

\section{Keywords:}

Community-acquired pneumonia

hospital treatment

outcome

prognostic analysis

Received: 29 August 1994

Accepted after revision 20 December 1994
An unchanged mortality of 4-21\% [1-3] in hospitaltreated community-acquired pneumonia (CAP) has renewed the interest in studying prognostic factors associated with fatal outcome. It is hoped that the knowledge of relevant prognostic factors might be useful for early identification of patients at high risk requiring intensive care treatment. This may, on the other hand, help to clarify the value of intensive care treatment for survival of severe CAP, a currently controversial issue [4-6].

Parameters correlated with mortality in univariate analysis have invariably failed to be of clinical value, mainly because of the large overlap making these parameters unreliable in an individual case. Multivariate analysis in a population fulfilling strict criteria of CAP was first applied by the British Thoracic Society (BTS) [7]. Three factors were found to be consistently correlated with death, respiratory rate $\geq 30$ breaths $\cdot \mathrm{min}^{-1}$, diastolic blood pressure $\leq 60 \mathrm{mmHg}$, and blood urea nitrogen $\geq 7 \mathrm{mmol} \cdot l^{-1}$ or mental confusion, and were included in a clinically applicable discriminant rule. The value of this rule as an objective instrument for clinical decision making may be questioned, because of the extreme cut-off values chosen for the parameters respiratory rate and diastolic blood pressure. It may simply qualify patients at risk of death, who apparently suffer from a severe clinical condition. Thus, the rule may result in redundant information.

Moreover, a recent study revealed a high rate of recurrence, and a mortality rate from pneumonia or any other cause twice as high as expected in an average follow-up period of 31 months after CAP, suggesting pneumonia as an index (or, less likely, to establish a new index) for another condition not yet fully understood [8]. It might, therefore, be prudent to base a prognostic analysis on a 
model that allows the weighing of covariates by the time until the event (i.e. death) occurs. The time of discharge from the hospital would be considered as censored observation. Such a model could prove more adequate in describing pneumonia as a dynamic event. It may also result in a more accurate judgement of relevant prognostic factors of the individual patient. We therefore performed a corresponding analysis in our patient population.

\section{Methods}

\section{Definitions}

Patients with CAP were retrospectively selected by review of the hospital records of patients with pneumonia between 1985-1993. Inclusion in the study population was based on the criteria of fever and a new radiographic infiltrate developing before admission to the hospital, and no other emerging diagnosis during treatment that replaced the initial diagnosis of CAP. All patients with pneumonia due to an obstructive tumour, tuberculosis, immunosuppressive diseases (with severe humoral or cellular immunodeficiency), and as an expected terminal event of other underlying end-stage diseases were excluded.

\section{Data collection}

Epidemiological, clinical, laboratory and microbiological data were extracted manually, recorded on study forms, and entered into a computerized data base. Overall, 54 independent variables were tested. These included the following: 1) data from history - cough, expectoration, haemoptysis, thoracic pain, dyspnoea, nausea, diarrhoea, cephalgia, myalgia, arthralgia, smoking and alcohol habits, and type and number of underlying diseases; 2) clinical data - sex, age, ages grouped from 15 up to 89 yrs with increments of 15 yrs, height, weight, body mass index, systolic, diastolic and mean arterial blood pressure, heart rate, and body temperature; 3) laboratory data - haemoglobin, leucocyte count, relative and absolute band neutrophils and lymphocytes, platelet count, aspartate aminotransferase (ASAT), alanine aminotransferase (ALAT), $\gamma$-glutamyltransferase (GGT), alkaline phosphatase (AP), bilirubin, total protein, albumin, lactate dehydrogenase (LDH), creatinine, urea nitrogen, and sodium, potassium; 4) capillary blood gases - oxygen tension $\left(\mathrm{PO}_{2}\right)$, carbon dioxide tension $\left(\mathrm{PCO}_{2}\right)$, calculated alveolar to arterial oxygen difference $\left(\mathrm{A}-\mathrm{aDO}_{2}\right)$, $\mathrm{pH}$, and base-excess; and 5) microbiological - established aetiology, specific aetiologies (S. pneumoniae, L. pneumophila, M. pneumoniae), and bacteraemia. All epidemiological and clinical data refer to the examination at admission. All laboratory data recorded were obtained within $24 \mathrm{~h}$ after admission. Range and consistency checks were performed on all data before the beginning of processing.

\section{Work-up of microbiological data}

All microbial investigations were performed according to clinical judgement. Pathogenic bacteria isolated from blood culture were considered definite evidence of aetiology. Serology consisted of a complement fixation (CF) test for Mycoplasma pneumoniae, Chlamydia psittaci, Coxiella burneti, Influenza A and B, Parainfluenza 1-3, respiratory syncytial virus (RSV) and adenoviruses, as well as an indirect immunofluorescence (IIF) test for Legionella pneumophila. A fourfold rise in immunoglobulin $\mathrm{G}$ ( $\mathrm{IgG}$ ) titre in a paired testing in any of the serological tests was considered definite evidence of infection. Single initial IgG or convalescent titres of $\geq 1: 80$ in the CF of Mycoplasma pneumoniae, C. psittaci, $C$. burneti or the viruses mentioned, or of $\geq 1: 256$ for $L$. pneumophila in the IIF were also classified as definite evidence of infection. Results of sputum cultures were not accepted as diagnostic, because no Gram stains were available for validation of sample qualities. Bronchoscopic examinations, including protected specimen brush and bronchoalveolar lavage (BAL) were performed in 25 pretreated patients. Because no standards are available for interpretation of positive cultures for pathogenic bacteria not achieving significant levels of growth in quantitative cultures after pretreatment, the results were discarded for analysis.

\section{Statistical analysis}

Descriptive statistics for continuous variables were expressed as the mean \pm the standard error of the mean (SEM). Differences in mean values between survivors and nonsurvivors were assessed using Student's twotailed t-test. The Chi-squared test was used for categorial variables, and Fisher's exact test in the case of small expected frequencies. Differences in mean values between survivors and nonsurvivors were assessed by a two factor analysis of variance (ANOVA) to correct for imbalance in sex distribution.

The univariate comparison of parameters of survivors and nonsurvivors, with survival time as dependent variable and an observation period censored by hospital discharge, was assessed using the Cox proportional hazards model. Factors found to be correlated with death in univariate analysis, with a level of significance less than $10 \%$, were entered into a multivariate backward stepwise selection model. A Chi-squared test was used for testing overall significance [9].

The three best parameters were included in a discriminant rule, by defining cut-off values and assigning one point for each parameter according to the cut-off value. The rule was tested by grouping cases with a score of two or more as nonsurvivors versus cases with less than two points as survivors.

In all cases, $p$-values of $<0.05$ were considered to be significant. Statistical analysis was performed using Statistical Package for the Social Sciences (SPSS) for Windows@. 


\section{Results}

\section{Description of patient population}

A total of 93 episodes in 92 patients represented the study population. Thirty two patients were treated in the Intensive Care Unit (ICU), including 13 requiring mechanical ventilation. Mean age was $51 \pm 19$ yrs (range 15-87 yrs). Sex distribution was 62 males and 30 females.

Forty two patients (45\%) had at least one underlying chronic disease as a risk factor for acquiring CAP (29 one, 10 two and three patients three). Of these, 21 patients had obstructive lung diseases, 2 fibrosing alveolitis, 10 congestive heart failure New York Heart Association (NYHA) II-IV, 7 chronic renal failure, 9 diabetes mellitus, 2 systemic autoimmune diseases (lupus erythematosus and scleroderma) and 7 disorders of the central nervous system (CNS) (five vascular, one amyotrophic lateral sclerosis, one epilepsy).

\section{Microbiology}

Microbial investigation was performed in 83 of the 93 cases $(89 \%)$. Nineteen investigations $(23 \%)$ were definitely diagnostic by blood culture or serology. The pathogens identified were: Streptococcus pneumoniae (six by blood culture); M. pneumoniae (five by serology); $L$. pneumophila (four by serology); Influenzavirus (two by serology); C. burneti (one by serology); Staphylococcus aureus (one by blood culture). Three pathogens, S. pneumoniae, M. pneumoniae and L. pneumophila accounted for 15 of the $19(79 \%)$ agents identified.

\section{Therapy}

Initial therapy consisted of a single antibiotic in 40 episodes and a combination regimen in 53 episodes. The most frequently used monotherapies were penicillins (11 patients), macrolides (11 patients), and fluoroquinolones ( 8 patients). Other agents prescribed included secondand third-generation cephalosporins (five patients), tetracyclines (three patients) and imipenem (one patient). The most frequently used combination was a broad-spectrum penicillin and second- or third-generation cephalosporins (27 patients). Other combination regimens included aminoglycosides (13), second- or third-generation cephalosporins (11), macrolides (8), broad-spectrum penicillins (6), fluoroquinolones (5), rifampicin (4), tetracyclines (3), fosfomycin (1) and aztreonam (1). The antibiotic regimen was changed at least once in 49 patients, and more than once in 19 patients. No general change in antibiotic prescription attitudes could be detected over the observation period.

\section{Mortality}

Twenty (22\%) of the patient population had a fatal outcome. Seven of these patients died within 7 days of admission, including two rapid deaths within $48 \mathrm{~h}, 6$ within 14 days and a further 3 within 28 days. Four patients died after four weeks of treatment. Eleven of the 12 patients requiring mechanical ventilation died. Four of the 19 (21\%) patients with a definite aetiological diagnosis had a fatal outcome (L. pneumophila (2), S. pneumoniae (1) and Influenzavirus (1)).

\section{Prognostic analysis}

Comparing survivors and nonsurvivors in univariate analysis, no significant differences could be found in age (50.3 \pm 19.1 versus $52.6 \pm 16.1)$, ascending age groups, sex (50/12 male versus 23/8 female), number or types of underlying diseases, established aetiology, specific aetiologies or bacteraemia. The significantly different values included seven parameters: mean values of heart rate, systolic, diastolic and mean blood pressure, percentage of lymphocytes, total protein and serum albumin. All remaining clinical and laboratory variables, including blood urea nitrogen, were not significantly different.

Using length of time spent in hospital as dependent variable in a univariate Cox regression hazard model, the four parameters systolic, diastolic and mean blood pressure, as well as percentage of lymphocytes, remained significant, whereas heart rate, total protein and serum albumin did not. The leucocyte count as well as LDH serum levels were added (table 1).

Table 1. - Results of univariate Cox hazard statistical analysis with length of survival time in hospital as dependant variable

\begin{tabular}{|c|c|c|c|c|}
\hline Variable & $\mathrm{n}$ & $\% \mathrm{NSu}$ & $\mathrm{Su}$ & $\mathrm{NSu}$ \\
\hline Heart rate $b \cdot \min ^{-1}$ & 89 & 21 & $98 \pm 2$ & $110 \pm 5$ \\
\hline Systolic BP mmHg & 82 & 20 & $127 \pm 3$ & $112 \pm 7 *$ \\
\hline Diastolic $\mathrm{BP} \mathrm{mmHg}$ & 82 & 20 & $74 \pm 2$ & $67 \pm 4 *$ \\
\hline Mean BP mmHg & 82 & 20 & $92 \pm 3$ & $82 \pm 4 * *$ \\
\hline Leucocytes $\cdot u 1^{-1}$ & 88 & 22 & $14158 \pm 760$ & $18453 \pm 2408 *$ \\
\hline Lymphocytes $\%$ & 81 & 20 & $15 \pm 1$ & $9 \pm 2 * *$ \\
\hline $\mathrm{LDH}^{\dagger} \mathrm{U} \cdot l^{-1}$ & 84 & 21 & $254 \pm 18$ & $399 \pm 4 * *$ \\
\hline
\end{tabular}

Results are expressed as mean \pm SEM. $\dagger$ : reference value 120-240 $\mathrm{U} \cdot l^{-1}$; Su: survivors; NSu: nonsurvivors; \% NSU: percentage of subjects being nonsurvivors. BP: blood pressure as measured by Riva-Rocci; LDH: lactate dehydrogenase; *, **: $\mathrm{p}<0.03,<0.02$ by Univariate Cox hazard $\mathrm{p}$ value.

Table 2. - Levels of significance in a multivariate backward stepwise Cox regression hazard model $(n=68)$

\begin{tabular}{lcc}
\hline Variable & $\beta$-coefficient & p-value \\
\hline Heart rate beats $\cdot \mathrm{min}^{-1}$ & 0.0311 & 0.0352 \\
SBP mmHg & -0.0173 & 0.1425 \\
LDH U. $l^{-1}$ & 0.0021 & 0.0154 \\
$\begin{array}{l}\text { Overall significance } \\
\text { of the model }\end{array}$ & Chi-squared & $\mathrm{p}$-value \\
Value & 12.864 & \\
\end{tabular}

SBP: systolic blood pressure; LDH: lactate dehydrogenase. 


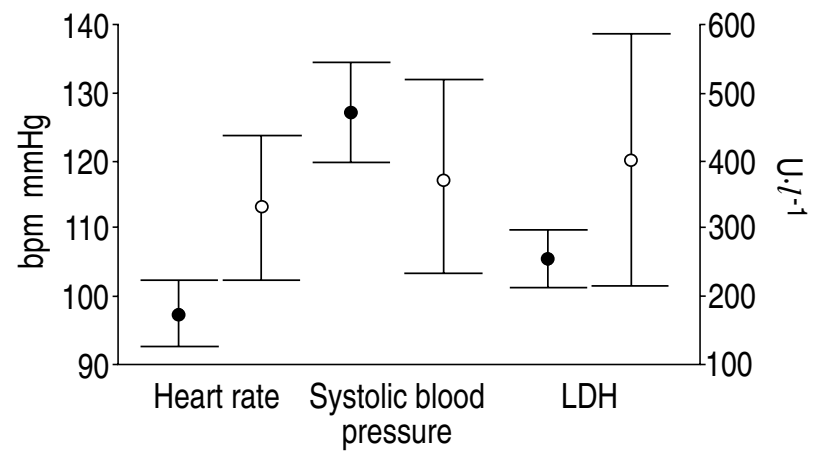

Fig. 1. - Mean values and SEM of the covariates heart rate (beats $\cdot \mathrm{min}^{-1}$ ), systolic arterial blood pressure $(\mathrm{mmHg})$ and lactate dehydrogenase $(\mathrm{LDH})\left(\mathrm{U} \cdot l^{-1}\right)$ in the Cox regression hazard function. bpm: beats $\cdot \mathrm{min}^{-1}$.

- : survivors; $\bigcirc$ : nonsurvivors.

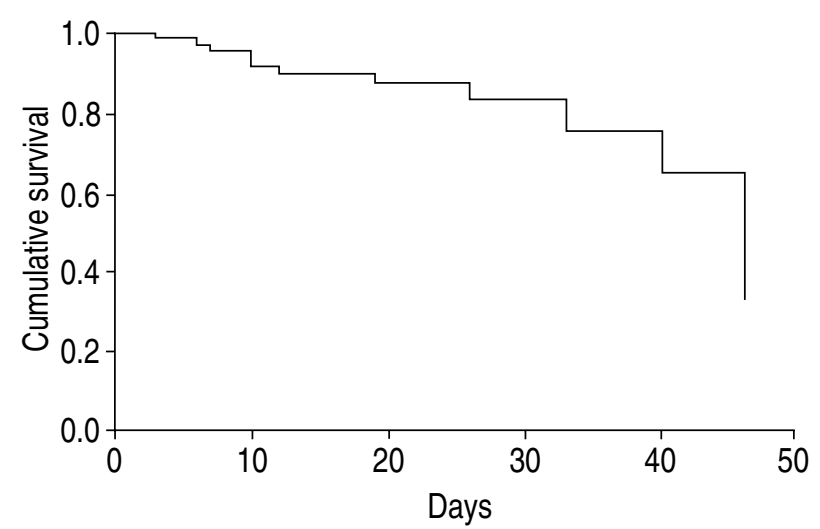

Fig. 2. - Cumulative survival function at means of covariates heart rate (beats $\left.\cdot \mathrm{min}^{-1}\right)$, systolic arterial blood pressure $(\mathrm{mmHg})$ and lactate dehydrogenase $(\mathrm{LDH})\left(\mathrm{U} \cdot l^{-1}\right)$.
Table 3. - Predicted outcomes of CAP using a discriminant rule consisting of two or more of the following: heart rate $\geq 90$ beats $\cdot \mathrm{min}^{-1}$, systolic arterial pressure $\leq 80$ $\mathrm{mmHg}$, and $\mathrm{LDH} \geq 260 \mathrm{U} \cdot l^{-1}$

\begin{tabular}{lrrrrrr}
\hline & \multicolumn{2}{c}{ Predicted } & \multicolumn{2}{c}{ Predicted } & \multicolumn{2}{c}{ Total } \\
& \multicolumn{2}{c}{ survivors } & \multicolumn{2}{c}{ nonsurvivors } & \multicolumn{2}{c}{ n } \\
& $\mathrm{n}$ & $\%$ & $\mathrm{n}$ & $\%$ & $\mathrm{n}$ & $\%$ \\
\hline Survivors & 41 & 75 & 14 & 25 & 55 & 81 \\
Nonsurvivors & 3 & 23 & 10 & 77 & 13 & 19 \\
Total & 44 & 65 & 24 & 35 & 68 & 100 \\
\hline
\end{tabular}

CAP: community-acquired pneumonia; LDH: lactate dehydrogenase.

These six variables were included in a multivariate backward stepwise selection analysis. Sixty eight sets of data were complete, including 13 sets of patients with fatal outcome (19\% mortality). The parameters found to contribute most to significance were heart rate, systolic blood pressure, and LDH values. Levels of significance are given in table 2. Figure 1 shows the mean values \pm SEM of the three parameters. Figure 2 shows the cumulative survival function at mean of the three covariates.

\section{Discriminant rule}

The variables significant in the multivariate analysis were also used to establish a discriminant rule. Optimal cut-off values were the following: heart rate $\geq 90$ beats $\cdot \mathrm{min}^{-1}$, systolic arterial pressure $\leq 80 \mathrm{mmHg}$,

Table 4. - Test performance of different discriminant rules in predicting mortality: comparison of reported studies with the present study

\begin{tabular}{|c|c|c|c|c|c|}
\hline Reference & $\begin{array}{c}\text { BTS } \\
\text { [7] }\end{array}$ & $\begin{array}{c}\text { Karalus } \\
\text { et al. } \\
{[11]}\end{array}$ & $\begin{array}{c}\text { FARR } \\
\text { et al. } \\
{[12]}\end{array}$ & $\begin{array}{c}\text { FARR } \\
\text { et al. } \\
{[12]}\end{array}$ & $\begin{array}{c}\text { Present } \\
\text { study }\end{array}$ \\
\hline Subjects $\mathrm{n}$ & 433 & 92 & 241 & 237 & 68 \\
\hline $\mathrm{Su} / \mathrm{NSu}$ & $409 / 24$ & $86 / 6$ & $221 / 20$ & $217 / 20$ & $55 / 13$ \\
\hline Deaths \% & 6 & 7 & 9 & 9 & 19 \\
\hline Age range yrs & $15-74$ & $13-97$ & $17-80$ & $17-80$ & $15-87$ \\
\hline Study period months & 13.5 & 10 & 25 & 25 & 108 \\
\hline Discriminant rule* & 1 & 1 or 2 & 1 & 2 & 3 \\
\hline Sensitivity $\mathrm{n}$ & $21 / 24$ & $5 / 6$ & $14 / 20$ & $7 / 20$ & $10 / 13$ \\
\hline$\%$ & 88 & 83 & 70 & 35 & 77 \\
\hline Specificity $\mathrm{n}$ & $322 / 409$ & $69 / 86$ & $186 / 221$ & $192 / 217$ & $41 / 55$ \\
\hline$\%$ & 79 & 80 & 84 & 89 & 75 \\
\hline PPV n & $21 / 108$ & $5 / 22$ & $14 / 49$ & $7 / 32$ & $10 / 24$ \\
\hline$\%$ & 19 & 23 & 29 & 22 & 42 \\
\hline NPV n & $322 / 325$ & $69 / 70$ & $186 / 192$ & $192 / 205$ & $41 / 44$ \\
\hline$\%$ & 99 & 99 & 97 & 94 & 93 \\
\hline Overall accuracy $n$ & $343 / 433$ & $74 / 92$ & $200 / 241$ & $199 / 237$ & $51 / 68$ \\
\hline$\%$ & 79 & 80 & 82 & 84 & 75 \\
\hline RR & 21 & 16 & 9 & 3 & 6 \\
\hline
\end{tabular}

*: discriminant rules - two or more variables of the following: respiratory rate $\geq 30$ breaths $\cdot \mathrm{min}^{-1}$; diastolic blood pressure $\leq 60$ $\mathrm{mmHg}$; blood urea nitrogen $\geq 7 \mathrm{mmol} \cdot l^{-1}$; (rule 1); the first two but with the presence of confusion instead of blood urea nitrogen (rule 2); heart rate $\geq 90$ beats $\cdot \mathrm{min}^{-1}$, systolic arterial blood pressure $\leq 80 \mathrm{mmHg}$; lactate dehydrogenase $\geq 260 \mathrm{U} \cdot l^{-1}$ (rule 3). BTS: British Thoracic Society; Su: survivors; NSu: nonsurvivors; PPV: positive predictive value; NPV: negative predictive value; $\mathrm{RR}$ : relative risk. 
and $\mathrm{LDH} \geq 260 \mathrm{U} \cdot l^{-1}$, resulting in a correct classification of survivors in $75 \%$, and of nonsurvivors in $77 \%$ of cases, respectively (table 3 ). The overall accuracy achieved by the rule was $75 \%$, the sensitivity $77 \%$, the specificity $75 \%$, the positive predictive value $42 \%$, and the negative predictive value $93 \%$. The relative risk (RR) was 6.1 (95\% confidence interval (95\% CI) 1.86-20.1) (table 4).

Seven of the 13 patients with fatal outcome required mechanical ventilation. When the discriminant rule was applied to this subgroup, all seven patients were correctly classified. Six patients who died were not intubated, and the rule correctly classified death in three.

\section{Discussion}

We found that heart rate, systolic blood pressure, and LDH serum levels were the variables most closely associated with fatal outcome in our multivariate analysis. Moreover, using these three parameters it was possible to establish a simple and clinically convenient discriminant rule.

Associations with fatal outcome that were confirmed as compared to other studies in the univariate Cox hazard analysis included decreased systolic, diastolic and mean blood pressures [7, 10-12]. Conversely, the prognostic significance of the mean leucocyte count, percentage of lymphocytes and LDH has not been established in previous reports. Heart rate [7], total protein, and serum albumin $[1,7,13]$ - parameters shown to be significant in other reports in univariate t-test analysis - did not remain significant in the univariate Cox hazard analysis calculation.

Whereas most previous reports showed age to be a significant prognostic factor $[1,2,5,14,15]$, we observed a mortality of $13-30 \%$ inconsistently distributed over the ascending clusters of age. In only one recent series on severe CAP [16], was age also not related to death. Moreover, in contrast to other studies [2, 15], we did not find the type or number of underlying diseases to be significantly associated with lethal outcome. The most likely explanation for these differences is the lack of a standardized approach to grading pre-existing and agerelated morbidity in a manner that makes these variables comparable.

As the number of definite aetiological diagnoses was relatively low, due to a "routine" microbial investigational approach, we could not confirm the prognostic significance of a specific pathogen, especially of Gramnegative agents, as found by others $[2,14,16]$. It is noteworthy, however, that two of four patients with $L$. pneumophila pneumonia died, despite appropriate therapy. This is consistent with reports on L. pneumophila as agents associated with increased mortality [5].

As we were interested primarily in prognostic factors which independently add prognostic information to the clinical judgement about the obvious current condition of the patient, we did not consider variables derived from intensive care treatment for our analysis. Thus, parameters which were found of prognostic significance in studies on severe CAP and which may imply severe or progressive disease in itself, such as mechanical ventilation, positive end-expiratory pressure (PEEP), fractional inspiratory oxygen $\left(\mathrm{FIO}_{2}\right)$, adult respiratory distress syndrome (ARDS) and type or number of complications $[6,14-16]$ were not included.

Because the respiratory rate was not recorded in our population, we were not able to compare the classification results to the rule established by the BTS [7]. Table 4 lists the results of the discriminant rules reported by the BTS, two studies validating this rule [11, 12], and our results. Besides the different statistical approach underlying the selection of parameters, the most important difference lies in the population studied. Our series included a higher relative number of deaths, thus indicating a population with more severe courses of CAP. Bearing this limitation in mind, our results appear comparable, despite a slightly minor overall accuracy rate. The positive predictive values were considerably higher in our population, conserving the high negative predictive values of the other studies. As the predictive values determine the applicability of a prognostic rule in clinical practice, our rule may contribute to a better selection of patients at risk of fatal outcome when validated in an independent patient cohort.

Criticism of the rule established by the BTS may address its clinical value, as parameters with extreme cut-off values were chosen. The respiratory rate and diastolic blood pressure as measured by Riva-Rocci can obviously prove sensitive only at the higher and lower extreme values, respectively. Moreover, as expected, the discriminant rule proved of limited value in patients with chronic renal failure and chronically elevated blood urea nitrogen levels [12]. The replacement of blood urea nitrogen for the variable of confusion - suggested as a possible alternative by the BTS - added a third variable that required extreme impairment by $\mathrm{CAP}$, and consistently lowered the sensitivity to $39 \%$ [7] and 35\% [12], respectively. This discriminant rule may, therefore, fail to contribute independent prognostic information to unarmed clinical judgement.

Our rule uses systolic rather than diastolic blood pressure, as a variable measured by sphygmomanometer (Riva-Rocci) more reliably, and heart rate as confident parameter at levels not necessarily indicating circulatory failure. The cut-off value of LDH also lies only slightly above the normal. Thus, our rule may prove of greater clinical value even with slightly minor classification results, as it includes parameters with cut-off values not obviously implying a severe clinical condition.

Limitations of our study may include patient selection, the relatively low absolute number of patients tested, and the limited number of patients with complete sets of data, due to the retrospective nature of the study. The patient population was derived from a tertiary care centre, thus, overrepresenting the number of hospitaltreated patients with severe CAP. This fact is reflected by the high mortality rate of $22 \%$. On the other hand, it is the population at highest risk, for which a prognostic analysis and a discriminant rule may prove most useful. Thus, the excellent predictive results achieved in 
the subgroup of mechanically-ventilated patients may be considered as further evidence for its potential value. Moreover, the high mortality resulted in a favourable proportion of patients for comparison in the relatively small absolute number of patients analysed.

In summary, we found heart rate, arterial systolic pressure and LDH to be best correlated with death in a multivariate analysis, and found a discriminant rule consisting of these three variables to achieve high predictive values. Thus, the rule qualifies for further prospective validation in an independent patient cohort of hospital-treated CAP.

\section{References}

1. Örtquist A, Hedlund J, Grillner L, et al. Aetiology, outcome and prognostic factors in patients with communityacquired pneumonia requiring hospitalization. Eur Respir $J$ 1990; 3: 1105-1113.

2. Marrie TJ, Durant H, Yates L. Community-acquired pneumonia requiring hospitalisation: 5 year prospective study. Rev Infect Dis 1989; 11: 586-599.

3. Fang GD, Fine M, Orloff J, et al. New and emerging etiologies for community-acquired pneumonia with implications for therapy: a prospective multicenter study of 359 cases. Medicine 1990; 69: 307-316.

4. Hook EW, Horton CA, Schaberg DR. Failure of intensive care unit support to influence mortality from pneumococcal bacteremia. JAMA 1983; 249: 1055-1057.

5. Woodhead MA, McFarlane JT, Rodgers FG, Laverick A, Pilkington R, Macrae AD. Aetiology and outcome of severe community-acquired pneumonia. J Infect 1985; 10: 204-210.

6. British Thoracic Society Research Committee and The Public Health Laboratory Service. The aetiology, management and outcome of severe community-acquired pneumonia on the intensive care unit. Respir Med 1992; 86: 7-13.
7. British Thoracic Society and the Public Health Laboratory Service. Community-acquired pneumonia in adults in British hospitals in 1982-1983: a survey of aetiology, mortality, prognostic factors and outcome. $Q \mathrm{~J} \mathrm{Med}$ 1987; 239: 195-200.

8. Hedlund JU, Örtquist AB, Kalin ME, Granath F. Factors of importance for the long-term prognosis after hospitaltreated pneumonia. Thorax 1993; 48: 785-789.

9. Dawson-Saunders B, Trapp RG. Basic and clinical biostatistics. Prentice-Hall International Inc., Appleton \& Lange, 1990.

10. Daley J, Jencks S, Draper D, Lenhart G, Thomas N, Walker J. Predicting hospital-associated mortality for medicare patients: a method for patients with stroke, pneumonia, acute myocardial infarction and congestive heart failure. JAMA 1988; 260: 3617-3624.

11. Karalus NC, Cursons RT, Leng RA, et al. Communityacquired pneumonia: aetiology and prognostic index evaluation. Thorax 1991; 46: 413-418.

12. Farr BM, Sloman AJ, Fisch MJ. Predicting death in patients hospitalized for community-acquired pneumonia. Ann Intern Med 1991; 115: 428-436.

13. Feldman C, Kallenbach JM, Levy H, et al. Communityacquired pneumonia of diverse etiology: prognostic features in patients admitted to an intensive-care unit and a "severity of illness" score. Intens Care Med 1989; 15: 302-307.

14. Moine P, Vercken JB, Chevret S, Chastang C, Gajdos $\mathrm{P}$, and the French study group for community-acquired pneumonia in intensive care unit. Severe communityacquired pneumonia: etiology, epidemiology, and prognosis factors. Chest 1994; 105: 1487-1495.

15. Pachon J, Prados MD, Capote F, Cuello JA, Garnacho J, Verano A. Severe community-acquired pneumonia: etiology, prognosis and treatment. Am Rev Respir Dis 1990; 142: 369-373.

16. Torres AJ, Serra-Batlles J, Ferrer A, et al. Severe community-acquired pneumonia: epidemiology and prognostic factors. Am Rev Respir Dis 1991; 114: 312318. 